\title{
THE CAUSE OF THE SPECTRAL TURNOVER IN THE GPS SOURCE 0108+388: FREE-FREE ABSORPTION OR SSA?
}

\author{
J. M. MARR \\ Union College, Schenectady, NY \\ F. CRAWFORD III \\ M.I.T., Boston, MA \\ AND \\ G. B. TAYLOR \\ N.R.A.O., Socorro, NM
}

The radio source $0108+388$ is a canonical example of a class of extragalactic radio sources, referred to as Gigahertz-Peaked Spectrum (GPS) sources, whose spectra peak at high frequencies. There are two competing models for the cause of the high frequency turnover: free-free absorption (f-f) of the lower frequency radiation by ionized gas in the host galaxies (e.g. van Breugel 1984), or synchrotron self-absorption (SSA) due to exceptionally large magnetic fields, (e.g. Hodges, Mutel, \& Phillips 1984).

We have initiated a study designed to distinguish between the two possibilities. Using VLBI observations over a range of frequencies we determine the apparent angular size of components as a function of frequency above and below the turnover. If the turnover is due to $\mathrm{f}-\mathrm{f}$ by line-of-sight gas, the angular size as a function of frequency below the turnover should be the same as above the turnover, but the source should get fainter at decreasing frequencies. If the turnover is due to SSA, though, then the source itself is opaque below the turnover and transparent above, and so $\theta$ vs. $\nu$ will differ noticeably on the two sides of the turnover.

We observed $0108+388$ with the VLBA at $22.2,8.4,5.0,2.3$, and 1.7 $\mathrm{GHz}$ on 24 June 1996 and at 15.3 and $8.4 \mathrm{GHz}$ on $03 \mathrm{Feb}$ 1997. Clean maps made from these data show flux-density distributions consistent with that found by previous investigators (e.g. Baum et al. 1990; Conway et al. 1994; Taylor, Readhead, \& Pearson 1996). We successfully modelfitted seven elliptical gaussian components to the data at the higher frequencies 
$(22,15$, and $8.4 \mathrm{GHz})$ and only four components at the lower frequencies, due to blending of the smaller components.

As noted by previous investigators, two major components consistently dominate the maps. For our purposes we need concentrate only on these major components, which we refer to as $\mathrm{C} 1$ and $\mathrm{C}$, following the notation of Taylor, Readhead, \& Pearson 1996. The flux-densities and angular sizes of these two components are listed in Table 1. As shown in the table, there appear to be significant jumps in the sizes of the components on the low frequency sides of the spectral peaks. This fits the scheme where the emission source is opaque below the peak and transparent above the peak, suggesting that SSA is indeed the cause for the turnover. The magnetic fields required in this case, following Kellerman \& Pauliny-Toth 1981, are $\approx 0.15$ Gauss in $\mathrm{C} 1$ and 0.25 Gauss in $\mathrm{C} 7$.

These results are not definitive, however, because the resolution at the longer-wavelengths is barely sufficient to properly delineate the smaller components from the major components. With the addition of a European antenna, though, an observation such as this would yield a definitive determination of the cause of the spectral turnover in this source; we therefore plan to re-observe with a global VLBI array.

This research was aided, in part, by funding from the Dudley Observatory. We are also grateful for the friendly assistance of the staff at the National Radio Astronomy Observatory.

TABLE 1. flux densities and major axes of the two major components in the best-fit models obtained with modelfitting.

\begin{tabular}{|c|c|c|c|c|}
\hline \multirow[t]{2}{*}{ Freq. $(\mathrm{GHz})$} & $\mathrm{F}_{\nu}(\mathrm{Jy})$ & major(mas) & $\mathrm{F}_{\nu}(\mathrm{Jy})$ & major(mas) \\
\hline & \multicolumn{2}{|c|}{ Component $\mathrm{C} 1$} & \multicolumn{2}{|c|}{ Component $\mathrm{C7}$} \\
\hline 1.663 & 0.222 & 2.14 & 0.045 & 3.49 \\
\hline 2.267 & 0.198 & 2.02 & 0.153 & 1.74 \\
\hline 4.983 & 0.302 & 0.72 & 0.240 & 0.60 \\
\hline 8.415 & 0.154 & 0.51 & 0.259 & 0.44 \\
\hline 15.349 & 0.115 & 0.53 & 0.152 & 0.47 \\
\hline 22.229 & 0.011 & 0.61 & 0.085 & 0.39 \\
\hline
\end{tabular}

\section{References}

Baum, S. A. et al. 1990, A\&A, 232, 19.

van Breugel, W. 1984, IAU Symp. 110, p. 59.

Conway et al. 1994, ApJ, 425, 568.

Hodges, M. W., Mutel, R. L., \& Phillips, R. B. 1984, AJ, 89, 1327.

Kellerman, K. I. \& Pauliny-Toth, I. I. K. 1981, Ann. Rev. Astr. Astrophys., 19, 373.

Taylor, G. B., Readhead, A. C. S., \& Pearson, T. J. 1996, ApJ, 463, 95 